\title{
Femmes au foyer et femmes en voyage.
}

Le cas des épouses des marchands assyriens au début du II ${ }^{\mathrm{e}}$ millénaire av. J.-C.

Housewives and travelling women: the wives of Assyrian merchants (early second millenium B.C.)

\section{Cécile Michel}

\section{(2) OpenEdition \\ Journals}

Édition électronique

URL : https://journals.openedition.org/clio/7603

DOI : 10.4000/clio.7603

ISSN : 1777-5299

Éditeur

Belin

Édition imprimée

Date de publication : 15 décembre 2008

Pagination : 17-38

ISSN : 1252-7017

Référence électronique

Cécile Michel, « Femmes au foyer et femmes en voyage. », Clio. Histoire, femmes et sociétés [En ligne], 28 | 2008, mis en ligne le 15 décembre 2011, consulté le 23 avril 2022. URL : http://

journals.openedition.org/clio/7603; DOI : https://doi.org/10.4000/clio.7603 


\section{Femmes au foyer et femmes en voyage. Le cas des épouses des marchands assyriens au début du $\mathrm{II}^{\mathrm{e}}$ millénaire av. J.-C.}

Cécile MICHEL

«Depuis un an, je n'ai rien fait d'autre que voyager dans des endroits reculés!», ainsi s'exprime la fille de l'un des plus célèbres marchands assyriens du début du IIe millénaire av. J.-C. à l'attention de son frère; malheureuse en affaires, elle lui demande de l'aide. Cette lettre a été découverte dans le comptoir de commerce de Kaniš, en Anatolie centrale (proche de la moderne Kayseri), avec 24000 tablettes cunéiformes paléo-assyriennes, qui constituent sans doute les plus abondantes archives privées du Proche-Orient ancien. Ces sources sont tout à fait exceptionnelles; elles documentent la vie quotidienne et les préoccupations de "femmes ordinaires», souvent oubliées des sources à la disposition des historiens ${ }^{1}$.

La masse la plus importante de textes cunéiformes est en effet composée par les archives officielles qui renseignent occasionnellement

1 La documentation cunéiforme exhumée au Proche-Orient ancien depuis le milieu du XIX ${ }^{e}$ s. couvre plus de trois millénaires d'histoire d'une région qui s'étend du golfe Arabo-persique à la Méditerranée et de l'Anatolie à l'Égypte. Elle est estimée aujourd'hui à environ un million de documents. Asher-Greve 2003 propose un point bibliographique sur la question des femmes dans le Proche-Orient ancien. Pour un état de la question sur la notion de genre en assyriologie, cf. Lion 2007. Sur les « femmes ordinaires », cf. plusieurs études publiées dans Parpola \& Whiting 2002, ainsi que Lion \& Michel 2006. 
divers aspects de la vie des reines et des grandes prêtresses ${ }^{2}$. Les archives privées, de leur côté, ne laissent guère de place aux femmes ; écrites pour et par des hommes, elles révèlent parfois la présence de femmes au foyer, à leurs côtés. Leurs activités économiques sont toutefois documentées par des contrats familiaux, des contrats d'achat de biens ou des prêts ${ }^{3}$.

Les femmes des marchands assyriens, tout comme leurs époux, ont laissé des lettres et des documents juridiques qui font allusion à divers voyages qu'elles ont accomplis, seules ou avec leur mari, et les motifs de ces déplacements. Certaines entreprennent le long voyage qui sépare Aššur, leur ville d'origine dans l'Irak actuel, de Kaniš, tandis que d'autres suivent leurs maris au cours de leurs déplacement en Asie Mineure. Les récits des voyages eux-mêmes sont rares ; le déroulement du voyage, souvent décliné au masculin, doit être reconstitué à partir d'allusions glanées dans les lettres.

\section{La société marchande paléo-assyrienne et ses archives}

Au début du $\mathrm{II}^{\mathrm{e}}$ millénaire av. J.-C., les habitants de la ville d'Aššur, sur le Tigre à une centaine de kilomètres au sud de l'actuelle Mossul, vivent essentiellement du commerce et développent des échanges intensifs avec l'Anatolie centrale et orientale. Ils s'installent en territoire étranger, à plus d'un millier de kilomètres de chez eux, pour vendre

2 Reines et grandes prêtresses ont fait l'objet de nombreuses études. Les premières se déplacent parfois pour rejoindre une cour étrangère dans le cadre d'un mariage diplomatique; elles voyagent rarement, par rapport au roi, et représentent même souvent leur époux absent à la cour. Voir par exemple les reines et princesses de Mari documentées par les archives du palais, Durand 2000: 259-504 et Ziegler 1999. Les grandes prêtresses demeurent dans le sanctuaire où elles ont été affectées. Pour la plus célèbre d'entre elles, Enheduanna, fille de Sargon d'Akkad, voir par exemple Winter 1987, Westenholz 1989 et McHale-Moore 2000.

3 Les archives des religieuses de la première dynastie de Babylone représentent une exception; mais ces femmes semblent particulièrement peu mobiles. Bien que très actives, elles délèguent à des hommes toute opération nécessitant une présence. Les mieux documentées, les naditum du dieu Šamaš à Sippar ont été étudiées par Harris 1975. 
l'étain et les étoffes qu'ils apportent avec eux. En retour, ils expédient à Aššur de l'or et de l'argent. Leur commerce est favorisé par des accords passés avec les princes anatoliens des différentes cités-États dans lesquelles ils ont organisé des comptoirs commerciaux ${ }^{4}$.

La ville d'Aššur est alors une cité-État indépendante, dominée par une oligarchie marchande ; elle s'étend sur une cinquantaine d'hectares et comprend quelques milliers d'habitants 5 . Le site n'a livré que très peu de documentation datée du début du $\mathrm{II}^{\mathrm{e}}$ millénaire car le quartier d'habitation paléo-assyrien n'a pas été retrouvé. Cette lacune de sources est heureusement comblée par les très nombreuses archives privées de marchands assyriens installés en Asie Mineure, principalement à Kanišs.

Les archives privées découvertes dans la ville basse du site de Kültepe, l'ancienne Kaniš, appartiennent principalement à trois ou quatre générations de marchands assyriens et à leurs familles; elles datent des XIXe et XVIII e siècles avant J.-C. ${ }^{6}$. Ces tablettes d'argile, inscrites en caractères cunéiformes et rédigées en dialecte paléoassyrien, étaient conservées dans des paniers, des jarres, des caisses et sur des étagères dans des pièces tenant lieu d'entrepôts de marchandises; cuites dans l'incendie qui détruisit plusieurs maisons de la ville, elles présentent un excellent état de conservation. La plupart des maisons du quartier commerçant de Kaniš, appelé kärum, ont fourni des lots d'archives dont la taille varie de quelques unités à plus d'un millier de textes ; quelques-uns relèvent d'autochtones qui, n'ayant pas de système d'écriture propre, ont emprunté syllabaire et dialecte paléo-assyriens.

4 Aššur figure au sud-est de la carte (Cahier d'images, figure 1) Les sites de Hahhum, Zalpa, Uršu, sur l'Euphrate, ainsi que Hurrama, Amkuwa et Hattuša comportent un comptoir de commerce assyrien. Au total, on dénombre une quarantaine de tels comptoirs en Asie Mineure ; celui de Kaniš tient lieu de centre administratif.

5 Cette période est appelée par convention «paléo-assyrienne ». Ce n'est qu'au XIVe siècle qu'Aššur devient le centre d'un véritable État territorial appelé Assyrie.

6 Bibliographie raisonnée des sources paléo-assyriennes, Michel 2003. Les lettres et les contrats familiaux analysés dans cet article ne sont pas datés; seules les créances portent une date. 
Un fonds d'archives comporte majoritairement des lettres expédiées depuis Aššur ou d'autres localités d'Asie Mineure, mais aussi quelques copies de lettres envoyées depuis Kaniš, des documents à valeur juridique (créances, contrats, procès-verbaux, verdicts de procès...) et des notices personnelles, essentiellement à caractère comptable ${ }^{7}$. Tous ces documents permettent de reconstituer la vie et les activités de leurs propriétaires, le système économique et politique des comptoirs de commerce assyriens en Anatolie, voire l'organisation de la société et les institutions de la ville d'Aššur d'où sont originaires les marchands assyriens.

L'exploitation de cette abondante documentation nécessite au préalable un important travail de prosopographie impliquant la distinction des nombreux homonymes, la reconstitution des couples et des familles, et l'identification des principaux lieux de résidence de chacun des individus.

Encouragés par des mesures politiques destinées à favoriser l'économie de la ville d'Aššur et par des conventions commerciales passées avec les gouvernements locaux, les marchands assyriens organisent des échanges à longue distance avec l'Asie Mineure ${ }^{8}$. Ils y exportent de l'étain provenant du Kazakhstan, d'Ouzbékistan et du Turkménistan ${ }^{9}$ et des étoffes achetées en Babylonie ou de confection locale ; au retour, ils rapportent à Aššur de l'or et de l'argent, en grande partie réinvestis dans de nouvelles caravanes. Ces échanges reposent sur un système complexe de prêts commerciaux, de partenariats à court ou long terme, de représentations mutuelles dans lesquels toute la société assyrienne est impliquée: dirigeants, clergé, notables et simples particuliers. La réussite de ce commerce international repose sur des firmes familiales dans lesquelles chacun des membres tient un rôle spécifique; basées à Aššur, elles ont des ramifications dans les différentes villes anatoliennes. Le père dirige l'entreprise depuis Aššur ;

7 La constitution des archives paléo-assyriennes a été étudiée en détail dans Veenhof 2003.

8 Pour une synthèse en français sur la période paléo-assyrienne avec la traduction de 400 lettres, cf. Michel 2001.

9 Cierny, Stöllner \& Weisberger 2005. 
il réunit les capitaux nécessaires à l'achat des marchandises et à l'organisation des caravanes, et gère les bénéfices acquis. Son fils aîné représente l'exploitation familiale à Kaniš ; il laisse parfois à Aššur femme et enfants en bas âge. Les autres garçons de la famille aident à la revente des marchandises en Asie Mineure, assurent les voyages entre les différents comptoirs de commerce et effectuent des allers et retours entre Aššur et Kaniš. Certains représentent la firme dans d'autres centres anatoliens où ils résident de manière plus ou moins temporaire.

Les femmes tiennent une place à part au sein de la firme. Les épouses des marchands assyriens, leurs filles et leurs servantes, tissent des étoffes servant d'une part à vêtir l'ensemble de la maisonnée et d'autre part à alimenter les exportations vers la Cappadoce ${ }^{10}$. En échange de leur production, appréciée pour la qualité du tissage, elles reçoivent de l'argent, voire de l'or, sous forme d'anneaux et de bijoux, destinés principalement à l'entretien de leur maison et de ses habitants ${ }^{11}$. Certaines de ces femmes restent à Aššur tandis que leurs maris, installés à Kaniš ou itinérants, s'organisent une nouvelle vie en Anatolie, avec une seconde épouse, souvent autochtone. Quelquesunes suivent leur époux à l'étranger. D'autres partent de leur propre chef pour se marier à Kaniš et accompagnent alors leur mari dans les différents comptoirs assyriens d'Asie Mineure. Ces divers cas sont documentés par les contrats familiaux (mariage, divorce) et par la correspondance échangée entre ces femmes et leurs maris, ou les hommes de leur famille.

\section{Voyager pour aller se marier}

Les contrats de mariage paléo-assyriens sont plutôt rares en regard du nombre de tablettes, sans doute parce que d'une part, le mariage standard n'implique pas un recours systématique à l'écrit, d'autre part, l'essentiel des mariages entre Assyriens devait avoir lieu à Aššur où l'on peut penser que les contrats, lorsqu'ils existaient, étaient conservés.

10 Veenhof 1972.

11 Michel 2006b. 
Dans ces contrats, le mariage est défini du point de vue de l'époux, la femme y intervient généralement comme objet. Même si cela n'est pas souvent explicite, on devine qu'il donne lieu à un transfert de biens entre les deux familles, suivi d'une cérémonie à la suite de laquelle la jeune fille rejoint la demeure de son mari ${ }^{12}$. La correspondance découverte à Kaniš reflète le désir constant des parents d'assurer le meilleur avenir possible pour leur fille : soit ils la consacrent à une divinité et lui laissent une part du patrimoine familial, soit ils lui choisissent un mari chargé de pourvoir à son entretien et elle reçoit une dot.

Le but premier du mariage en Mésopotamie ancienne est d'assurer une descendance, héritière du patrimoine familial, qui prend en charge l'entretien des parents âgés et le culte des ancêtres décédés. Par conséquent, en cas de stérilité, la législation autorise le mari à prendre une deuxième femme pour procréer; cette dernière n'a pas le statut d'épouse. Mais la condition très particulière des marchands, absents du foyer conjugal pendant de très longues périodes, installés dans des comptoirs de commerce loin de chez eux en Asie Mineure, les autorise à choisir une deuxième épouse sur place. Il s'agit là d'une particularité propre aux marchands assyriens: leur mode de vie, centré sur le voyage, les a introduit à pratiquer une sorte de bigamie. Cette tradition tient compte en effet de la situation géographique éclatée de la famille, et distingue deux types d'épouses: la principale (aššatum), souvent assyrienne, se trouve généralement à Aššur, tandis que l'épouse secondaire (amtum), plutôt anatolienne, demeure en principe en Anatolie. Par conséquent un homme peut avoir deux épouses, mais ne vit qu'avec une seule en même temps ${ }^{13}$.

Pour respecter au mieux cette règle, les Assyriens prêtent une attention toute particulière au lieu de célébration de leur premier mariage, lieu qui ne correspond pas nécessairement à la ville d'origine des futurs époux. Deux lettres envoyées par des marchands à leurs fils insistent pour que ceux-ci viennent se marier à Aššur; dans les deux

12 Lion \& Michel 2006.

13 Michel 2006a. 
cas, la femme et son futur mari, qui résident l'un et l'autre dans une localité d'Asie Mineure, doivent entreprendre le long voyage vers Aššur. Les villes d'Aššur et de Kaniš sont éloignées d'environ $1300 \mathrm{~km}$, et les caravanes mettent environ six semaines pour parcourir cette distance.

La première missive est adressée par Aššur-imittī à son fils Ușur-šaIštar $^{14}$ :

À propos de ton épouse (aššatum), j’ai écrit ceci à Aššur-bēl-awātim et à toi-même : 'Si tu veux l'épouser et venir (ici, à Aššur) avec son père, alors épouse-la. Si tu ne viens pas (ici), ne l'épouse pas.' Maintenant, si ŠūKūbum (doit arriver) le premier, qu'elle vienne avec lui, si toi tu es là avant, qu'elle vienne avec toi.

Le père de la jeune femme, qui doit assister à la cérémonie, doit également venir à Aššur.

La seconde lettre, émanant d'Irišum à l'attention de Dān-Aššur, délivre à peu près le même message ${ }^{15}$ :

Si le fils de Panaka te dit ceci : 'Épouse la jeune fille !' Alors (réponds-lui) ainsi: '(Selon) les instructions de mon père, je vais me rendre à la Ville (Aššur) ; envoie-la à la Ville (Aššur) et je l'épouserai dans la Ville (Aššur).' Je t'en prie, ne l'épouse pas là-bas.

Dans les deux cas, la future mariée doit donc quitter sa ville de résidence, sans doute Kaniš, pour aller se marier et vraisemblablement demeurer à Aššur, chez sa belle-famille. Les fiancés n'effectuent pas nécessairement le voyage de concert ; la jeune fille est accompagnée de son père, voire d'un autre homme de son entourage.

Certaines femmes semblent toutefois réticentes à l'idée de voyager pour aller s'installer dans une localité qui leur est inconnue. L'une d'elle, Nuhšātum, devait épouser Puzur-Aššur dans Kaniš ; mais ce

$14 \mathrm{Kt} \mathrm{n/k} \mathrm{1138,} \mathrm{1.} \mathrm{1-16;} \mathrm{références} \mathrm{et} \mathrm{édition} \mathrm{en} \mathrm{français} \mathrm{dans} \mathrm{Michel} \mathrm{2006a:} \mathrm{169,}$ n. 53.

15 Kt 83/k 164, l. 1-17; références et édition en français dans Michel 2006a: 169, n. 54. Dans les deux cas, il est probable que des considérations d'ordre juridique (l'application du droit assyrien) ou financier motivent cette décision. 
dernier ayant déménagé à Wahšušana, environ 200 km à l'ouest, écrit à sa promise l'enjoignant de le retrouver pour l'épouser ${ }^{16}$ :

Ton père a écrit chez moi à ton sujet pour que je t'épouse. Et moi, j’ai alors envoyé mes serviteurs et ma lettre chez ton père à ton sujet pour qu'il te laisse partir. Je t'en prie, le jour où tu prendras connaissance de ma tablette, tourne-toi vers ton père (pour qu'il y consente) et mets-toi en route avec mes serviteurs. Je suis seul, il n'y a personne qui me serve, ni personne qui dresse la table pour moi! Si tu ne devais pas venir avec mes serviteurs, alors j'épouserai dans Wahšušana une jeune fille wahšušanéenne ! Fais-y attention, et toi et mes serviteurs, ne vous attardez pas, partez!

Puzur-Aššur s'adresse directement à sa future épouse, et non au père de cette dernière auprès duquel il convient toutefois d'obtenir le consentement. Ses serviteurs doivent escorter la jeune femme entre Kaniš et Wahšušana, et sans doute également assurer le transport de ses effets personnels, dont sa dot. Si Nuhšātum refuse de partir et rejoindre Puzur-Aššur, alors ce dernier se sent dégagé de toute obligation et libre de se choisir une autre épouse.

Ces différents exemples semblent indiquer que la jeune femme encore célibataire ne voyage pas seule, mais en compagnie d'un homme, vraisemblablement chargé d'assurer sa protection durant le trajet.

\section{Femmes au foyer et sédentaires.}

\section{Les assyriennes à Aššur, les Anatoliennes à Kaniš}

Puzur-Aššur, qui menace sa fiancée d'épouser une autochtone si elle ne le rejoint pas rapidement, prend pour prétexte la solitude qu'elle lui impose par son absence. Cette solitude est le lot quotidien des femmes d'Aššur, délaissées par leurs maris partis exercer leurs activités commerciales en Asie Mineure. De fait, au début du XIX siècle, les premières générations de marchands assyriens qui partent vendre étain et étoffes laissent épouses et enfants à Aššur pour des périodes de plus

16 BIN $6104=$ Michel $2001: \mathrm{n}^{\circ} 397$. Cette lettre non seulement fait une allusion tout à fait exceptionnelle aux tâches domestiques des épouses, mais également témoigne d'une certaine expression des sentiments de la part du fiancé. 
en plus longues. Aux six semaines du voyage vers Kaniš, s'ajoutent celles nécessaires au dédouanement des marchandises auprès des autorités anatoliennes, aux transactions sur place pour leur vente au comptant ou à crédit, à l'organisation des convois avec l'or et l'argent et au voyage de retour vers Aššur. Dès l'ouverture des routes à la fin de l'hiver, les premières caravanes partent pour l'Asie Mineure; les marchands effectuent en moyenne deux voyages aller et retour chaque année, ils sont donc absents de chez eux environ huit mois par an. Certains d'entre eux restent plus longtemps à Kaniš, voire s'installent sur place pour diriger la branche anatolienne de la firme familiale.

Cette longue séparation des époux a généré une correspondance féminine, découverte à Kaniš, tout à fait exceptionnelle, qui témoigne des activités de ces épouses livrées à elles-mêmes à Aššur. Là, elles accomplissent toutes les tâches de la femme au foyer, devenue chef de famille, et représentent les intérêts financiers de leurs maris auprès des collègues ou des autorités assyriennes de la ville. Ces femmes élèvent seules les plus jeunes enfants du couple et leur assurent éducation et subsistance; elles entretiennent leurs domestiques, plus ou moins nombreux selon leur niveaux de vie. Pour l'achat de la laine et des céréales, elles disposent de l'argent envoyé par leurs époux ou issu du produit de la vente de leurs étoffes. Leur correspondance témoigne également de leurs activités pieuses, offrandes et prières aux dieux et aux esprits des morts, et des leçons de morale qu'elles donnent à leurs compagnons.

Quelques-unes se plaignent de la solitude qui leur est imposée par les activités professionnelles de leur mari dont elles réclament le retour à Aššur :

Je t'en prie, lorsque tu auras entendu (ma) tablette, viens, regarde vers Aššur, ton dieu et ton domaine, et tant que je vivrai, que je puisse voir tes yeux (te voir en personne). 
Tarām-Kūbi n'a toutefois pas été exaucée, car son mari décède en Anatolie ${ }^{17}$. De même, Lamassī meurt seule à Aššur, tandis que son époux Pūšu-kēn se trouve encore à Kanišs ${ }^{18}$. D'autres femmes, plus heureuses, retrouvent leur mari après une séparation de plusieurs années, voire de plusieurs dizaines d'années ${ }^{19}$.

Ces femmes n'ont pas d'autre solution que d'attendre le retour de leurs maris auprès d'elles. En revanche, pendant leur séjour prolongé en Asie Mineure, certains marchands, comme cela a déjà été indiqué, contractent un second mariage sur place. Les archives concernant cette seconde épouse, souvent une autochtone, ont également été retrouvées à Kaniš ; elles comportent les lettres expédiées par leurs maris alors qu'ils voyagent dans les différents comptoirs de commerce d'Asie Mineure, ou séjournent à Aššur. Les Anatoliennes, restent dans leurs maisons à Kaniš où elles ont des activités assez semblables aux femmes d'Aššur ${ }^{20}$ : entretien des domestiques, éducation des enfants, surveillance de la maison et des marchandises qu'elle renferme. En revanche, elles ne sont pas toujours seules, car leur maison abrite souvent d'autres membres de la famille et elles accueillent régulièrement des associés de leurs maris. Ces femmes, qui résident en général dans leur ville d'origine, ne voyagent guère car parmi leurs tâches quotidiennes figurent des travaux agricoles qui les retiennent à la maison. Elles élèvent des bœufs de labour et des porcs et achètent

17 Pour Tarām-Kūbi, femme d'Innāya, cf. Matouš 1982 : 268-270, Michel 1991 :77-88 et $2001: 464-470$.

18 Lamassī, femme de Pūšu-kēn, Veenhof 1972 : 111-118, Michel 2001 : 425-438.

19 Imdīlum, après 25 années passées en Anatolie, s'est retiré des affaires et est retourné à Aššur. Selon Ichisar 1981, Larsen 1982 et Michel 2001: 464-470, la femme d'Imdīlum, Šìmat-Aššur, serait restée tout ce temps à Aššur. Toutefois, selon Kryszat 2007, l'épouse d'Imdīlum se nomme Ištar-bāšti (tout comme sa fille) et elle aurait vécu un temps à Kaniš.

20 Cf. par exemple Hatala, la fille d'Enišaru et épouse aššatum de Laqēpum cf. Veenhof 1978 et Michel 2001 : 501-502 ; Šišahšušar, l'épouse amtum d'Aššur-nādā, Larsen $2002: \mathrm{n}^{\circ} 50$, cf. également les lettres $\mathrm{n}^{\circ} 51-58$, ainsi que $\mathrm{n}^{\circ} 73$ qui serait une copie d'une lettre de Šišahšušar à son mari et Michel 2001 : 476-480. 
divers outils nécessaires aux travaux des champs ${ }^{21}$. Lorsqu'un marchand assyrien décide de se retirer des affaires et retourne vivre à Aššur, il quitte son épouse anatolienne ; la séparation est formalisée par un contrat de divorce 22 .

\section{Des femmes qui voyagent avec leurs époux : les Assyriennes en Anatolie}

Contrairement aux Anatoliennes, qui paraissent peu mobiles, il semble que les Assyriennes d'Asie Mineure soient amenées à se déplacer fréquemment. Certaines ont fait le long voyage depuis Aššur, d'autres sont nées sur place, souvent de couples mixtes. Dans le contrat passé à Kaniš qui les lient à leur époux assyrien, il est stipulé qu'elles doivent accompagner leur mari dans tous ses déplacements en Anatolie, et que ce dernier n'est pas autorisé à se remarier dans une autre localité munie d'un comptoir commercial; cette clause figure essentiellement dans les contrats liant un marchand originaire d'Aššur à une Assyrienne ayant le statut d'amtum 23 :

Aššur-malik a épousé Suhkana, la fille d'Irma-Aššur. Partout où Aššurmalik se rendra, il l'emmènera avec lui. Il ne prendra pas d'autre (épouse) dans Kaniš, ni n'installera (une autre femme) à ses côtés. Il n'épousera ni une fille d'Aššur ni une Anatolienne. S'il en épouse une, alors Aššur-malik devra verser 5 mines d'argent à Suhkana. (En revanche), dans la ville d'Aššur, il épousera la fille de Dada. (Témoins).

Cette restriction à deux femmes, l'une dans le pays d'origine, la seconde dans le pays d'adoption, est destinée à protéger le statut des

21 Larsen 2002 : xxix ; Michel 2006b : 171-172 et 2008. Hamananika représente une exception dans la mesure où il est prévu, dans son contrat de mariage : "Aššurmalik n'épousera pas une autre femme aššatum dans Kaniš, Burušhattum, Durhumit ou Wahšušana ; il emmènera son épouse aššatum (env.)/amtum (tab.) là où il le voudra. », TPAK 1161.

22 Plusieurs contrats précisent les modalités de cette séparation lorsqu'il y a des enfants : le père peut emmener ses enfants avec lui après avoir versé une indemnité de divorce et les frais de bouche et d'éducation des enfants ou il laisse les enfants, tout particulièrement les filles, à leur mère.

23 Kt 94/k 149 publié par Michel \& Garelli 1996. 
deux épouses. La polygamie est interdite : le marchand n'est pas autorisé à prendre une épouse dans chaque ville qu'il visite!

Les lieux mentionnés dans les contrats, susceptibles d'être l'objet de la visite du marchand, couvrent toute la zone concernée par leur commerce $^{24}$ :

Puzur-Ištar a pris pour épouse (amtum) Ištar-lamassī, fille d'Aššur-nādā. Il l'emmènera à Burušhattum ou dans le Hattum partout où ses déplacements (le mèneront) ; et il devra la ramener avec lui à Kanišs.

Le terme géographique Hattum désigne la région comprise à l'intérieur de la boucle du Kiz1lırmak, et Burušhattum, non encore identifiée, correspond à la localité la plus occidentale fréquentée par les Assyriens. Néanmoins, dans une lettre que lui adresse son mari, on apprend qu'Ištar-lamassī n'est pas de tous les voyages ${ }^{25}$ :

Là-bas, surveille attentivement toute la maison... Je t'en prie, ne t'en vas pas quelque part à l'extérieur jusqu'à ce que tu entendes des instructions précises de ma part.

Dans la pratique il y a donc un compromis : l'épouse n'accompagne pas systématiquement son mari mais demeure parfois à domicile pour la gestion des affaires courantes.

Les lettres font fréquemment allusion aux voyages des marchands, et plus rarement de leurs épouses ${ }^{26}:$ "Tu m'as dit ceci : 'prends ta femme et viens'». Lorsque le couple s'absente, il abandonne temporairement sa maison où sont entreposées marchandises et archives ; pour protéger ses biens des voleurs, il s'assure de la présence d'un proche. Aššur-rabi et Hattītum ont ainsi laissé leur maison de Kaniš aux bons soins de leur fille Maganika alors qu'ils effectuent un déplacement en Asie Mineure, mais cette dernière semble avoir confié

24 Prag I 490, 1-10; références et édition en français dans Michel 2006a : 162, n 25, 166 , n 45 . Plus généralement sur les différentes localités mentionnées dans les contrats de mariage, p. 165-166.

25 VS 26, 53 traduit par Michel $2001: \mathrm{n}^{\circ} 362$.

26 BIN 6, 52, 7'-9'. 
la bâtisse à un tiers au grand dam de ses parents qui lui reprochent son attitude ${ }^{27}$ :

Pourquoi laisses-tu demeurer dans la maison un inconnu tandis que toi, tu es partie pour (vivre) chez un homme? Si tu (es) ma fille, surveille attentivement la maison, nous arriverons dans dix jours!

La femme qui accompagne son mari lors de ses voyages professionnels s'assure qu'il lui reste fidèle et qu'il lui procure de quoi être nourrie et logée; il s'agit là de l'une des obligations de l'époux ${ }^{28}$. Cette coutume rompt aussi la solitude du marchand, perpétuellement sur les routes; elle est cependant plus difficile à mettre en pratique lorsque le couple a des enfants.

En outre, lorsque les déplacements s'avèrent très fréquents, ce mode de vie itinérant n'est pas toujours du goût de l'épouse, parfois amenée à voyager seule pour rejoindre son mari. Ištar-nādā se plaint d'une telle situation dans la première page d'une lettre pathétique qu'elle expédie à Inna-Sîn alors momentanément à Kaniš ; on ne sait pourquoi elle ne voyage pas avec lui, mais elle tente à plusieurs reprises de le rejoindre, sans succès ${ }^{29}$ :

Tu m'as laissée dans Burušhattum, et vraiment je suis sortie de l'esprit de mon mari. Tu ne prends même pas soin de moi! Je suis venue et dans Kaniš tu m'as dénigrée, déshonorée et tu ne m’as pas laissée venir dans ton lit ! Tu m'as écrit ainsi depuis Timilkiya : 'Si tu ne viens pas ici, tu n'es plus mon épouse-(amtum)! Je t'ai montrée du soir au matin à ceux de Burušhattum.' Depuis Timilkìya, tu es ensuite allé à Kaniš et tu (m’as dit) ceci : 'Je (re)partirai dans 15 jours.' Au lieu de 15 jours, tu (y) es resté une année ! Depuis Kaniš tu m’as écrit ainsi: 'Monte à Hahhum !' Voilà une

27 BIN 6, 20 traduit par Michel 2001: n³34. Hattītum voyage également sans son mari selon une lettre qu'elle expédie à Zikri-elka: «Depuis que je suis allée à Wahšušana, j'ai eu cinq fois (l'occasion de) me mettre en colère!», BIN 6, 93 traduit par Michel $2001: \mathrm{n}^{\circ} 333$.

28 S'il n'entretient pas son épouse, son contrat peut-être annulé ; TC 1 67: «En outre, s'il n'arrive pas dans les deux mois et ne prend pas soin de sa femme, on donnera la jeune fille à un autre époux »; références et édition en français dans Michel 2006a : 162, n 22.

$29 \mathrm{Kt} \mathrm{h} / \mathrm{k} 73$; références et édition en français dans Michel 2006a : 170, n 56. Cette lettre est conservée au Musée d'Ankara (Cahier d'images, figure 2). 
année que je demeure à Hahhum et dans tes envois, tu ne mentionnes même pas mon nom! Tes représentants qui demeurent là-bas ont pu voir mes jours (s'écouler, seule).

Ištar-nādā prétend obéir aux ordres de son époux mais ne parvient pas à le retrouver, à chaque fois qu'elle arrive quelque part, il est déjà reparti dans une autre localité. Depuis Burušhattum, à l'ouest, elle se rend à Kaniš puis sur l'Euphrate à Hahhum; elle ne donne malheureusement aucune information relative aux conditions de ses voyages et de son hébergement.

\section{Des femmes qui voyagent seules}

Il apparaît toutefois qu'Ištar-nādā se déplace seule et, à la lecture des lettres découvertes à Kaniš, cela semble être le lot de nombreuses femmes. Certaines ont entrepris le long voyage qui sépare Aššur de Kaniš, d'autres visitent les différents comptoirs de commerce d'Asie Mineure, quelques-unes enfin, plus rares, ont parcouru la route qui mène de Kaniš à Aššur.

Une ou deux générations après la mise en place des comptoirs de commerce assyriens en Asie Mineure, quelques femmes d'Aššur effectuent le long voyage entre Aššur et Kaniš et décident même parfois de s'installer sur place. Un exemple d'un tel éclatement familial est donné par les archives exhumées en 1991 dans la maison d'Elamma ${ }^{30}$. La fille aînée d'Elamma, Ummī-Išhara, consacrée au dieu Aššur, écrit depuis la ville homonyme à sa sœur Šalimma qui se trouve à Kaniš, afin de lui donner des conseils pour sauver son couple. En effet, Šalimma a laissé mari et enfants à Aššur, pour se rendre auprès de sa mère, déjà veuve, et refuse de revenir à Aššur malgré les courriers répétés de son époux. Ummī-Išhara met sa sœur en garde : frustré de ne pas voir son épouse, son mari risque de demander le divorce, elle rapporte les propos qu'il lui a tenus ${ }^{31}$ :

30 Ces archives sont étudiées par K.R. Veenhof qui en prépare la publication. Pour la situation de Šalimma, cf. Veenhof 2007.

31 Texte Kt 91/k 385 édité par Veenhof 2007. 
À plusieurs reprises, je lui ai écrit une lettre, mais elle refuse de venir ici. Que pourrais-je lui envoyer qui surpasserait les messages qui lui sont déjà parvenus plusieurs fois ? (...) Parce qu'elle refuse de venir, tu ne dois plus me parler (...) Parce qu'elle ne se comporte pas comme mon épouse (amtum) et qu'elle refuse de venir ici, tu ne dois plus mentionner son nom devant moi au risque de ne plus être ma sœur.

Ummī-Išhara conseille donc à sa sœur :

Pourquoi d'autres dirigent-ils tes enfants et ta maison alors que toi tu restes là-bas ? (...) Si tu vois une possibilité pour venir, mets-toi en route et viens ici avant que l'homme ne change d'avis. Le jour où Pilah-Ištar est arrivé ici, comme tu n'étais pas venue avec lui, il était très malheureux et n'a pas quitté sa maison pendant cinq jours! Si tu cherches un autre époux, écris-moi afin que je le sache! Sinon, mets-toi en route et viens ici!

Cette lettre présente donc le cas d'une femme mariée qui abandonne sa famille à Aššur pour rejoindre sa mère à plus d'un millier de kilomètres, et y reste contre l'avis de son époux.

En revanche, c'est par amour pour son épouse-amtum, Ištar-ummī, demeurée à Aššur, qu'Aššur-taklāku, qui vit avec son épouse principale en Asie Mineure, lui écrit depuis Kanišs ${ }^{32}$ :

Si tu m'aimes vraiment, mets-toi en route et viens. Cette femme que j’ai épousée intrigue contre toi (...) Ne manque pas de partir rapidement et monte ici! Ne me ruine pas! mets-toi en route et viens par la prochaine (caravane)!

Sa femme est donc censée voyager avec la caravane de marchandises. Toutefois cette belle lettre, conservée dans son enveloppe intacte, n'est jamais partie, elle est restée dans les archives de l'expéditeur!

32 Texte RA 51, 6 traduit par Michel $2001:$ n³96. Ce texte présente un cas atypique où l'épouse secondaire est assyrienne et habite Aššur. En effet, après quelques générations, de plus en plus d'Assyriens s'installent en Anatolie; ils amènent leur femme avec eux, font venir des jeunes filles d'Aššur pour les épouser ou encore prennent pour femme des autochtones. Certains ont en effet choisi pour épouse principale une femme de Kaniš et se retrouvent avec une épouse secondaire à Aššur. 
Certaines femmes, vraisemblablement veuves ou divorcées, ont décidé de leur propre chef de s’installer à Kaniš et s'investir dans l'entreprise familiale sur place. Parmi celles-ci figure la fille d'Imdīlum, Ištar-bašti ${ }^{33}$; sans doute déjà veuve de son premier mari, $\bar{A} l$-țāb ${ }^{34}$, elle écrit à son frère Puzur-Ištar ${ }^{35}$ :

Viens rapidement afin que je puisse partir avec toi, et (ainsi) je pourrai surveiller la maison de ton père et la tienne dans Kaniš, et personne ne créera d'ennuis à la maison de votre père.

Ištar-bāšti ne conçoit pas de voyager seule ; elle attend l'arrivée de son frère afin de partir avec lui. Une fois installée à Kaniš, elle reçoit des instructions de son père quant à la gestion de ses affaires. Elle finit par se remarier avec un autochtone au grand mécontentement de son père, retourné vivre à Aššur, qui a dû lui verser une seconde fois une $\operatorname{dot}^{36}$.

Parmi toutes les lettres mentionnées ci-dessus, seule celle adressée par Aššur-taklāku à son épouse secondaire Ištar-ummī fait allusion au mode du voyage: elle doit venir avec la caravane de marchandises. C'est certainement le cas le plus classique pour les longs déplacements entre Aššur et Kaniš ; rejoindre une caravane permet de profiter de la logistique mise en place pour les nuitées dans les relais et surtout de bénéficier d'une protection contre brigands et pillards. Les voyages

33 Cette famille a été étudiée par Ichisar 1981 et Larsen 1982. Selon Kryszat 2007, cette fille, Ištar-baštī (II) aurait été adoptée par Imdīlum et par sa femme Ištar-baštī (I) ; elle se nommerait également Zizizi.

34 Voir la lettre Prag I 688 republiée par Kryszat 2007 : 211-213 et qui aurait été envoyée par Ištar-bāšti à ses parents alors en Asie Mineure.

35 KTS 1, 1b traduit par Michel $2001: \mathrm{n}^{\circ} 352$.

36 Texte VS 26, 33 traduit par Kryszat 2007 : 213-215. Cette situation n'est pas unique, en effet, les archives exhumées en 1994 à Kaniš présentent un cas similaire ; elles sont étudiées par Larsen s. p. Šalim-Aššur est le plus jeune des fils d'Issu-rik. Marié à Ennam-Ištar, il a deux filles : Lamassī, femme consacrée résidé à Aššur, Šāt-Anna vit en Anatolie et fut mariée à deux reprises; la seconde fois elle a épousé un Anatolien nommé Šuppi-numman. 
s'effectuent souvent à pied, les bagages voyageant à dos d'âne ${ }^{37}$. Il semble que toute famille devait avoir un âne. Ne pas posséder un âne apparait comme un signe de pauvreté, comme en témoigne cette lettre émanant d'une femme ${ }^{38}$ :

Que donnerai-je aux enfants? Que donnerai-je à notre mère ? Que dirai-je pour ma nourriture ? Ici je n'ai pas même un âne à monter!

Plusieurs messages font allusion au voyage à dos d'âne et il existe des selles en cuir pour ânes. Les marchands les plus aisés préfèrent se déplacer en Anatolie à dos de mule: le pas est plus agréable et cet hybride est beaucoup plus rapide que l'âne ${ }^{39}$. Le transport des enfants, sur de courtes distances, a lieu en carriole tractée par un âne ; Lamassī explique à son époux ${ }^{40}$ : «Comme la fillette avait grandi, j’ai dû faire une paire d'étoffes épaisses pour la carriole ». Cependant, l'essentiel des témoignages sur les modes de déplacement et les étapes dans les relais émanent d'hommes; les femmes ne donnent aucun détail sur le déroulement de leurs voyages.

Quel que soit le mode de transport choisi, les conditions de voyage ne sont pas toujours faciles, les routes sont mauvaises et impraticables en hiver; les témoignages de décès d'ânes en cours de route sont monnaie courante. On peut comprendre que certaines femmes aient été réticentes à l'idée d'entreprendre un long voyage pour s'installer et vivre en pays étranger.

De nombreuses Assyriennes, et plus rarement quelques Anatoliennes, effectuent des déplacements en Asie Mineure pour divers motifs ; leurs lettres précisent les localités qu'elles ont visitées mais pas le mode de transport utilisé lors de leur déplacement et la durée de

37 Pour l'organisation des voyages en général, cf. Michel sous presse; pour les différents modes de transport, cf. Veenhof 1972 : 1-12 ; Michel 2004 ; Dercksen 2004 : 255-285.

38 BIN 6 183, 9-17.

39 Michel 2004.

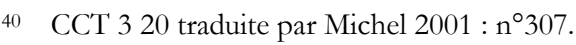


celui-ci. Ennam-Ištar, l'épouse de Šalim-Aššur, écrit à son fils ainé alors que son époux est décédé ${ }^{41}$ :

En ta présence, dans la ville de Burušhattum, j’ai donné 10 sicles d'argent à Aššur-tāb. Dans Kaniš, je lui ai donné une seule étoffe-kutānum.

Tout comme Ištar-nādā (cf. ci-dessus), Šišahšušar qui tient sa maison à Kaniš espère pouvoir rejoindre son mari installé temporairement dans un autre comptoir de commerce d'Asie Mineure ; cependant l'hiver s'est installé, les routes sont impraticables et son mari lui conseille d'attendre quelques mois ${ }^{42}$ :

Pour ce que je t'avais écrit, à savoir : 'Je t'écrirai au début du printemps, et tu viendras ici', ne te mets pas en colère! L'hiver nous a surpris et les capitaux d'exploitation que je possède sont maintenant convertis en marchandises à crédit, voilà pourquoi je ne t’ai pas écrit (plus tôt).

Lorsqu'il ne s'agit pas de rejoindre leur époux, les femmes voyagent pour régler des affaires commerciales ou familiales, parfois sur les injonctions d'un homme de leur famille, telle Hašušarna qui reçoit les instructions suivantes d'Aššur-taklāku, peut-être son mari, et d'IddinŠamaš 43 :

Le jour où tu prendras connaissance de notre message, mets-toi en route et viens à Tegarama. Dans Tegarama, supervise mes biens jusqu'à ce que je passe. Qu'un mot de toi me parvienne (pour m'indiquer le) jour où tu penses partir (pour moi). Ensuite va, et offre [’’argent] dans Wahšušana.

Partir en voyage demande un minimum d'organisation et Kunnanīya, qui doit rejoindre son mari, reçoit les instructions suivantes de sa part :

(Fais des achats) puis mets-toi en route. (...) Laisse Šāt-Aššur (fille du couple) aux bons soins de l'esclave Walawala (...) dès que la situation dans le comptoir de commerce sera apaisée, mets-toi en route. Šamaš-taklāku doit dormir à la porte (pour surveiller la maison).

41 Texte Kt 94/k 614 publié par Larsen s. p. : n²23.

42 Texte CCT 37 a traduit par Michel $2001: n^{\circ} 361$ et Larsen $2002: n^{\circ} 50$.

43 Texte HUCA 40, 55 traduit par Michel $2001: n^{\circ} 395$. 
Kunnanīya est l'une des rares Anatoliennes à se rendre à Aššur, mais seulement après le décès de son mari, afin d'obtenir une part dans sa succession auprès de sa belle-famille; en son absence, elle confie sa maison de Kaniš à sa sœur aînée et à son frère, mais son voyage est un échec ${ }^{44}$.

Les quelques femmes qui voyagent seules sont donc fréquemment des femmes mariées et leurs déplacements sont souvent suggérés par leurs époux. Quelques-unes cependant, telle Šalimma, partent contre l'avis de leur mari, tandis que d'autres, veuves, prennent elles-mêmes l'initiative de partir en voyage. Les motivations de ces dernières sont d'ordre économique ou familial.

Les premières générations d'Assyriens qui s'aventurent en Asie Mineure sont des hommes, vraisemblablement déjà mariés dans leur ville natale. Leur femme assyrienne, demeurée seule à Aššur, gère leur maisonnée. Installés loin de chez eux pour des périodes de plus en plus longues, ces Assyriens contractent sur place un second mariage, souvent avec une autochtone. Ces marchands épousent donc deux types de femmes qui ont, en définitive, des vies assez semblables : les Assyriennes d'Aššur et les Anatoliennes de Kanišs. Sédentaires par rapport à leur mari, elles doivent conserver précieusement marchandises et archives et représentent ses intérêts. Les Anatoliennes exécutent en plus des travaux agricoles qui les retiennent à la maison.

Quelques générations plus tard, la situation a évolué. Quelques Assyriennes, souvent veuves, ont entrepris le grand voyage qui sépare Aššur de Kaniš dans le but de s'installer en Asie Mineure. Là, tout comme les Assyriennes nées ou arrivées très jeunes à Kaniš, elles épousent Assyriens ou autochtones. Dans le premier cas, selon leur contrat de mariage, elles doivent suivre leur mari dans tous ses déplacements sur le plateau anatolien; de même que leurs époux, elles sont donc fréquemment sur les routes et s'organisent, en leur absence, pour protéger leur maison et les biens qu'elle renferme contre les voleurs ou contre banquiers et associés mécontents qui seraient tentés

44 Michel 1998 et 2001 : 493-499. 
de venir se servir. Ces couples se sont parfaitement adaptés à leur nouvel environnement; leurs maisons à Kaniš présentent une architecture et un mobilier typiquement anatolien, et on peut imaginer qu'il en va de même pour les divers pied-à-terre dont ils disposent dans les différents comptoirs de commerce d'Asie Mineure. Bien qu'elles soient prêtes à mener une vie souvent itinérante, quelques femmes ont toutefois des difficultés à tenir le rythme des voyages imposé par leurs époux, et se contentent de les rejoindre dans certaines localités.

L'immigration croissante des hommes et des femmes d'Aššur en Asie Mineure accélère la mixité des populations des différentes citésÉtats anatoliennes : de plus en plus, au sein d'une même famille, frères et sœurs portent des noms d'origines ethniques différentes.

Les archives des marchands de Kaniš, de nature essentiellement commerciale, permettent de reconstituer certains aspects de la vie quotidienne de leurs auteurs parmi lesquels figurent les voyages entrepris par les hommes et les femmes entre Aššur et l'Asie Mineure ou dans les différents comptoirs de commerce anatoliens. Mais certaines facettes de ces déplacements échappent à l'écrit ; ces sources de la pratique, lettres et contrats, ne proposent guère de récits de voyage. Tels qu'ils sont ils n'en constituent pas moins une source exceptionnelle sur les marchands et leurs épouses du début du deuxième millénaire avant notre ère.

\section{Bibliographie}

Asher-Greve Julia M., 2003, "Women and Gender in Ancient Near Eastern Cultures: Bibliography 1885 to 2001 AD », NIN. Journal of Gender Studies in Antiquity, III, p. 33-114.

Cierny Jan, StÖllner Thomas \& Gerd Weisberger, 2005, "Zinn in und aus Mittelasien », in Ünsal YalçıN, Cemal Pulak \& Rainer SlotTa (Hrsg.), Das Schiff Uluburun - Welthandel vor 3000 Jahren (Catalogue d'une exposition à Bochum du 15 juillet 2005 au 15 juillet 2006), Bochum, Deutsches Bergbau-Museum, p. 431-448.

Dercksen Jan Gerrit, 2004, Old Assyrian Institutions, Leyde, Publications de l'Institut historique-archéologique néerlandais de Stamboul, vol. XCVIII (MOS Studies IV). 
Durand Jean-Marie, 2000, Documents épistolaires du palais de Mari, tome III, Paris, Éditions du Cerf, coll. «Littératures anciennes du Proche-Orient », XVIII.

HARrIS Rivkah, 1975, Ancient Sippar: A Demographic Study of an Old Babylonian City (18941595 B. C.), Leyde, Publications de l'Institut historique-archéologique néerlandais de Stamboul, vol. XXXVI.

ICHISAR Metin, 1981, Les archives cappadociennes du marchand Imdillum, Paris, Édition ADPF, coll. « Recherches sur les grandes civilisations », 3.

KrysZat Guido, 2007, «Eine Frau mit Namen Zizizi», Altorientalische Forschungen, XXXIV, p. 210-218.

LARSEN Mogens Trolle, 1982, "Your Money or your Life! A Portrait of an Assyrian Businessman ", in J. Nicholas Postgate (ed.), Societies and Languages of the Ancient Near East, Studies in Honor of I. M. Diakonoff, Warminster, Aris \& Phillips Ltd, p. 214-245.

—, 2002, The Ašsur-nada Archive, Leyde, Nederlands Instituut voor het nabije Oosten (Old Assyrian Arcbives 1).

—, sous presse, The Archive of the Šalim-Aššn Family, vol. I: the first two generations, Anakra, Ankara Kültepe Tabletleri, Türk Tarih Kurumu.

LiON Brigitte, 2007, « La notion de genre en assyriologie », in V. SÉBILLOTTE CuCHET et N. Ernoult (éd.), Problèmes du genre en Grèce ancienne, Paris, Publications de la Sorbonne, p. 51-64.

Lion Brigitte \& Cécile MiCHEL, 2006, «As mulheres em sua família : Mesopotâmia, $2^{\circ}$ milênio a.C. ", Tempo, XIX (Rio de Janeiro), p. 149-173.

Matouš Lubor, 1982, «Zur Korrespondenz des Imdilum mit Taram-kubi », in G. Van DrIEL et al. (ed.), Zikir sumim. Assyriological Studies Presented to F.R. Kraus on the Occasion of this Seventieth Birthday, Leyde, p. 268-270.

McHale-Moore Rhonda, 2000, "The Mystery of Enheduanna's Disk », Journal of the Ancient Near Eastern Society, XXVII, p. 69-74.

Michel Cécile, 1991, Inn ya dans les tablettes paléo-assyriennes, Paris, Éditions Recherche sur les Civilisations, ADPF, 2 vol. : I. Analyse, II. Edition des textes.

—, 1998, «Les malheurs de Kunnanīya, femme de marchand », Archivum Anatolicum, III (1997), Ankara, 1998, p. 239-253

—, 2001, Correspondance des marchands de Kaniš au début du II ${ }^{e}$ millénaire av. J.-C., Paris, Éditions du Cerf, coll. «Littératures anciennes du Proche-Orient», XIX.

—, 2003, Old Assyrian Bibliography of Cuneiform Texts, Bullae, Seals and the Results of the Excavations at Assur, Kültepe/Kanis, Acemböyük, Alishar and Bogazköy, Leyde, Publications de l'Institut historique-archéologique néerlandais de Stamboul, XCVII (Old Assyrian Archives Studies 1). 
—, 2004, "The perdum-mule, a mount for distinguished persons in Mesopotamia during the IInd millennium B.C. ", in Barbara S. FrIZELL (ed.), Man and animal in Antiquity, Rome, Acta Instituti Romani Regni Sueciae, p. 194-204 .

—, 2006a, «Bigamie chez les Assyriens du début du II millénaire », Revue Historique de Droit Français et Étranger, LXXXIV, p. 155-176.

—, 2006b, «Femmes et production textile à Aššur au début du II e millénaire avant J.C. », Techniques \& culture, XLVI, p. 281-297.

—, 2008, «Les Assyriens et leurs femmes anatoliennes », in Jan GerRit DerCKSEN (ed.), Anatolia and the Jazira during the Old Assyrian Period, Leyde, Publications de l'Institut historique-archéologique néerlandais de Stamboul (Old Assyrian Archives Studies, III), p. 209-229.

—, sous presse, «Les pérégrinations des marchands assyriens en haute Mésopotamie et en Asie Mineure », Res Antiquae, V.

Michel Cécile \& Paul Gareldi, 1996, "New Old Assyrian Marriage Contracts", Anadolu Medeniyetleri Müzesi ynlliğ (1995), Ankara, p. 295-302.

Parpola Simo \& Robert M. Whiting, 2002, Sex and Gender in the Ancient Near East, Proceedings of the XLVII Rencontre Assyriologique Internationale, Helsinki, The NeoAssyrian Text Corpus Project, Institute for Asian and African Studies.

VeEnhof Klaas R., 1972, Aspects of the Old Assyrian Trade and its Terminology, Studia et Documenta ad Iura Orientis Antiqui Pertinentia, vol. x, Leyde.

—, 2003, Archives of Old Assyrian Traders ", in M. Brosius (ed.), Archives and Archival Tradition. Concepts of Record Keeping in the Ancient World, Oxford, p. 78-123.

-, 2007, "Sisterly Advice on an Endangered Marriage in an Old Assyrian Letter », in Martha T. ROTH, Walter FARBER, Matthew W. STOLPER \& Paula von Bechtolsheim (eds), Studies Presented to R.D. Biggs, June 4, 2004, Chicago, The Oriental Institute of the University of Chicago, From the Workshop of the Chicago Assyrian Dictionary, vol. 2 (Assyriological Studies, XXVII).

Westenholz Joan G., 1989, «Enheduanna, En-Priestess, Hen of Nanna, Spouse of Nanna ", in H. BeHrens, D. Loding \& M. Roth (ed.), Dumu é.dub.ba.a. Studies in Honor of A. Sjöberg, Philadelphie, University Museum, p. 539-556.

WiNTER Irene, 1987, «Women in Public: the Disk of Enheduanna, the Beginning of the Office of EN-Priestess and the Weight of Visual Evidence », in J.-M. DurAND (éd.), La femme dans le Proche Orient antique. Actes de la XXIII rencontre assyriologique internationale, Paris, 1986, Paris, ERC, p. 189-202.

ZIEGLer Nele, 1999, Le Harem de Zimrî-Lîm, Florilegium marianum, IV (Mémoires de NABU, V). 